\title{
ARTICLE OPEN \\ Satisfaction with a digitally-enabled telephone health coaching intervention for people with non-diabetic hyperglycaemia
}

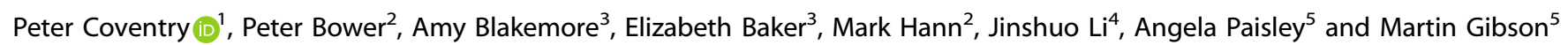

International evidence shows that lifestyle interventions can effectively reduce the risk of developing diabetes in people with nondiabetic hyperglycaemia (NDH). A candidate intervention that has potential to be rolled out at population level is health coaching. Digital interventions offer the means to potentially enhance user satisfaction with health coaching and improve efficiencies. We used a randomised controlled trial to test whether a digitally-enabled health coaching intervention that included an online dashboard and telephone health coaching improved user satisfaction and cost-efficiencies compared with a telephone only health coaching intervention. The primary outcome was satisfaction measured by Client Satisfaction Questionnaire (CSQ-8). 103 participants with NDH were allocated to the telephone coaching only intervention and 106 participants with NDH were allocated to the digital and telephone coaching intervention. In an intention-to-treat analysis satisfaction was higher in participants allocated to the digital and telephone coaching intervention than those allocated to the telephone only intervention, but the difference was not significant. There were no significant differences between the groups on secondary outcomes (HbA1c, BMl, activation, depression, self-management, health status). From a service commissioning perspective the mean incremental cost of the digitally-enabled intervention was $£ 236$ ( $\$ 332 ; € 270$ ). Call times, including administration, were longer for participants allocated to the digitallyenabled intervention. The results show that user satisfaction with digitally-enabled intervention is broadly equivalent with that of telephone delivered interventions in the context of routinely delivered diabetes prevention programmes. There is scope for future work that assesses how economies of scale can be achieved at larger user bases.

npj Digital Medicine (2019)2:5; https://doi.org/10.1038/s41746-019-0080-6

\section{INTRODUCTION}

Diabetes is a major global public health threat affecting nearly half a billion adults. ${ }^{1}$ Over $90 \%$ of cases are type 2 diabetes which develops in the presence of genetic, environmental and behavioural factors; the majority of cases are attributable to excess body weight and sedentary lifestyles. ${ }^{2}$ Unmanaged, type 2 diabetes is associated with serious microvascular and macrovascular complications that can lead to significant disability and premature mortality. ${ }^{3}$ The cost implications to health systems is considerable: the total cost of diabetes care in the UK National Health Service (NHS) was $£ 23.7$ billion ( $\$ 33.8$ bn, $€ 27.4$ bn) in 2010-11, with a projected annual cost of $£ 39.8$ bn ( $\$ 56.8$ bn, $€ 46$ bn) by $2035-36 .^{4}$

The onset of these complications can occur during a latent or pre-diabetic phase characterised by impaired glucose metabolism with fasting plasma glucose levels below internationally agreed thresholds for diabetes. Non-diabetic hyperglycaemia (NDH) is a high-risk category of developing diabetes and is a term used to describe pre-diabetic conditions associated with decreased ability of the body to regulate glucose effectively, such as impaired glucose regulation (IGR) and impaired fasting glucose and/or impaired glucose tolerance. ${ }^{5}$

There is unequivocal evidence that lifestyle interventions can reduce the risk of developing diabetes. ${ }^{6}$ When targeted at people with $\mathrm{NDH}$, lifestyle interventions that promote physical activity, diet modification and weight loss can reduce the risk of diabetes by $58 \%{ }^{7,8}$ However, while the use of lifestyle interventions to prevent diabetes in people with $\mathrm{NDH}$ are recommended in national guidelines, they are usually resource intensive to deliver and are unlikely to be cost-effective and implementable at scale across routine settings. ${ }^{9}$

Identifying cost-effective lifestyle interventions that can be deployed in routine settings to prevent diabetes in high risk populations such as those with NDH is therefore a health policy priority. ${ }^{10}$ Health coaching that includes modelling behaviour and goal setting, has emerged as a promising candidate platform that can support the delivery of effective lifestyle interventions for people with long term conditions, ${ }^{11}$ including diabetes ${ }^{12}$ and possibly people with $\mathrm{NDH} .{ }^{13}$ With the flexibility of being accessible by telephone, health coaching can potentially reach a significant proportion of the target population.

It is possible that reach and efficiency of telephone health coaching could be enhanced further by digitally-enabled components. The last decade has seen increased use and availability of second generation web-based interventions that include program and interactive content, multimedia materials and guidance and feedback. ${ }^{14}$ Web-based interventions have been especially used to promote behaviour change and manage mental health problems. ${ }^{15,16}$ And there is good evidence that technology assisted primary care interventions that combine either the internet,

\footnotetext{
${ }^{1}$ Department of Health Sciences and Centre for Reviews and Dissemination, University of York, York, UK; ${ }^{2}$ NIHR School for Primary Care Research, Centre for Primary Care, Manchester Academic Health Science Centre, University of Manchester, Manchester, UK; ${ }^{3}$ Division of Nursing, Midwifery and Social Work, School of Health Sciences, University of Manchester, Manchester, UK; ${ }^{4}$ Department of Health Sciences, University of York, York, UK and ${ }^{5}$ Salford Royal NHS Foundation Trust, Salford, UK Correspondence: Peter Coventry (peter.coventry@york.ac.uk)
}

Received: 7 June 2018 Accepted: 21 November 2018

Published online: 04 February 2019 


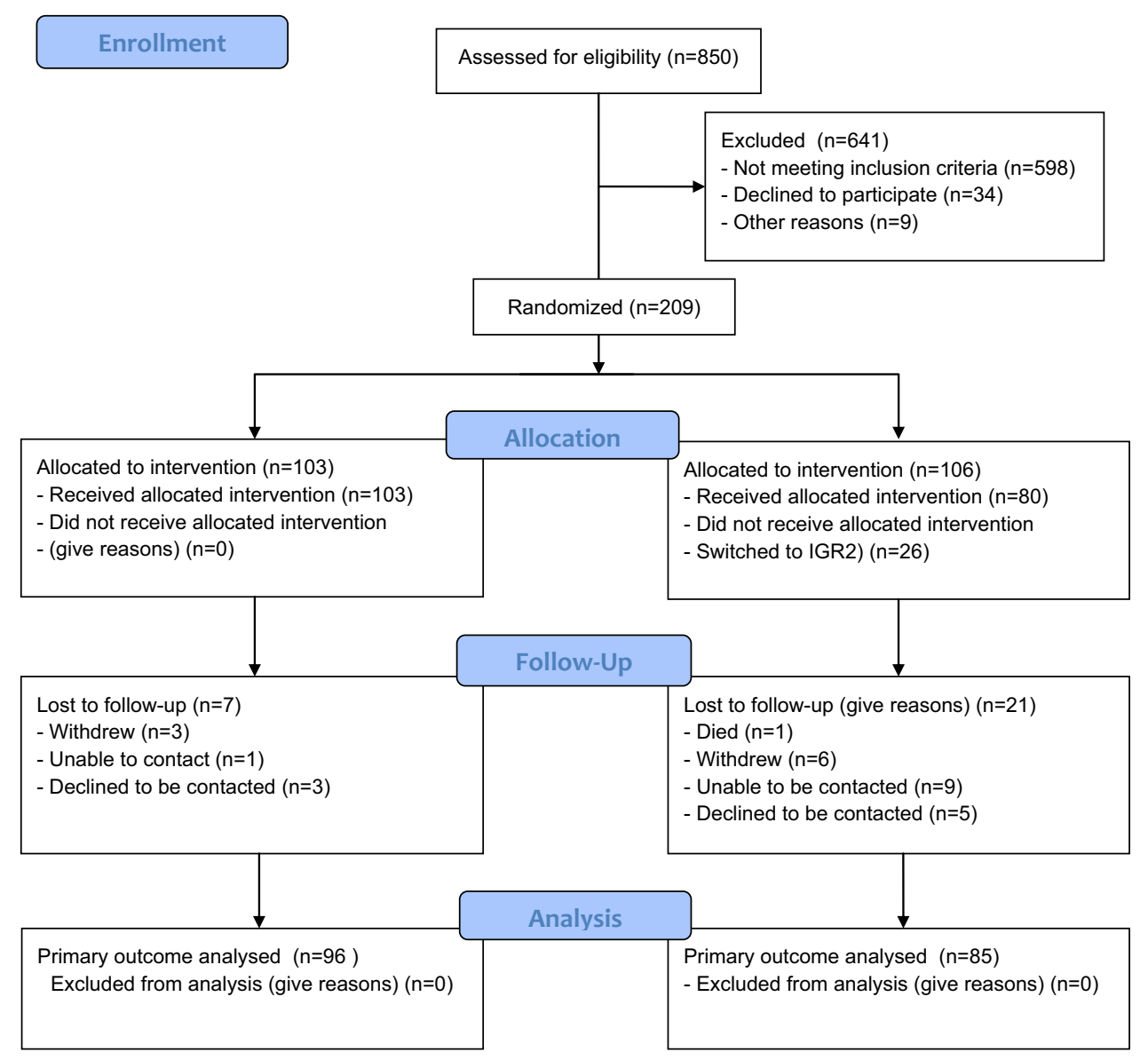

\section{Fig. 1 CONSORT flow diagram}

personal computer, and/or a mobile device are effective in supporting people to lose weight. ${ }^{17}$ Fully automated interventions that support web-based and mobile facilitated goal setting and personalised behaviour change have also proven effective in reducing cardiovascular risk among overweight and obese adults and in improving glycaemic control and reducing other risk factors for diabetes, but gains are short-lived. ${ }^{18,19}$

While there is an emerging consensus about best practice in developing and evaluating effective web-based interventions, there is less emphasis on understanding how end-user satisfaction can inform successful implementation in routine settings. ${ }^{20}$ In the context of web-based interventions user satisfaction and experience ratings are important metrics that go beyond developer assessments of design and build quality and potentially provide critical feedback about usability and acceptability relevant to healthcare providers. ${ }^{21}$ Satisfaction is increasingly used as an outcome of interest in evaluations of web-based health interventions and there is growing recognition that satisfaction can capture user perception of their experience of web-based interventions. $^{22,23}$

In the context of the CATFISH trial a telephone-only health coaching intervention for people with $\mathrm{NDH}$ was established as routine care and the aim of the trial was therefore to compare two active treatments to determine if a digitally-enabled health coaching intervention improved user experience and led to higher efficiency. Specifically the CATFISH trial aims to assess whether a digitally-enabled telephone coaching intervention (IGR3) is more acceptable than an existing telephone-only coaching intervention (IGR2) for people with NDH. Secondary objectives of the trial are to (1) determine whether the delivery of the IGR3 intervention is more efficient than the existing commissioned IGR2 intervention; (2) to explore the costeffectiveness of IGR3 in comparison with IGR2; (3) to qualitatively explore and compare user and provider experience of IGR3 and IGR2 interventions; and (4) to explore the impact, if any, of IGR3 compared with IGR2 on clinical outcomes relevant to diabetes prevention in people with NDH

\section{RESULTS}

Participant recruitment and retention

Between July 2015 and May 2016 there were 853 referrals from general practice in Salford to Care Call. Of these 253 (29.7\%) verbally consented to be contacted by the research team at the University of Manchester about participating in the CATFISH trial. Of those that consented to be contacted 210 (83\%) agreed to take part in the CATFISH trial and were assessed for eligibility and invited to a baseline assessment following receipt of their written informed consent. The first participant was recruited on 30th June 2015 and the last participant was recruited on 25th May 2016. One participant was withdrawn from the trial before allocation because they were found to have type 2 diabetes. 209 participants were randomised, with 103 allocated to IGR2 and 106 allocated to IGR3. $87 \%$ of participants returned a follow-up questionnaire for the primary outcome. The flow of participants is shown in Fig. 1.

\section{Baseline characteristics of participants}

Participants had a mean age of 58.3 (SD 11.4) years and $44 \%$ were female. The majority (94\%) were from white ethnic backgrounds and just under half $(47.5 \%)$ were either in full or part-time paid employment. Participants had a mean HbA1c of 44.4 (SD 1.6) 
Table 1. Baseline characteristics of participants

\begin{tabular}{|c|c|c|}
\hline & IGR2 $(n=103)$ & IGR3 $(n=106)$ \\
\hline Mean age & $59.0(11.3)$ & $57.6(11.6)$ \\
\hline \multicolumn{3}{|l|}{ Age in categories: } \\
\hline $25-44$ years & $9(8.7 \%)$ & $11(10.3 \%)$ \\
\hline $45-64$ years & $59(57.3 \%)$ & $64(60.4 \%)$ \\
\hline $65-84$ years & $35(34.0 \%)$ & $31(29.2 \%)$ \\
\hline Sex (female) & $50(48.5 \%)$ & $42(39.6 \%)$ \\
\hline \multicolumn{3}{|l|}{ Ethnicity } \\
\hline White (British//rish/Other) & 97 (94.2\%) & 99 (93.4\%) \\
\hline Non-White & $6(5.8 \%)$ & $7(6.6 \%)$ \\
\hline \multicolumn{3}{|l|}{ Economic status } \\
\hline Working full-time & $34(33.3 \%)$ & $41(39.4 \%)$ \\
\hline Working part-time & $13(12.7 \%)$ & $11(10.6 \%)$ \\
\hline Unemployed & 7 (6.9\%) & $3(2.9 \%)$ \\
\hline Permanently sick & $8(7.8 \%)$ & $8(7.7 \%)$ \\
\hline Retired & $34(33.3 \%)$ & $32(30.8 \%)$ \\
\hline $\begin{array}{l}\text { Other (F/T Education/Looking after } \\
\text { Home/Something Else) }\end{array}$ & $6(5.9 \%)$ & $9(8.6 \%$ \\
\hline Missing data & 1 & 2 \\
\hline Mean BMI & $33.4(6.7)$ & $34.4(8.0)$ \\
\hline Mean $\mathrm{HbA} 1 \mathrm{c}(\mathrm{mmol} / \mathrm{mol})$ & $44.7(1.6)$ & $44.2(1.6)$ \\
\hline Mean patient activation (PAM-13) & $62.2(14.3)$ & $60.8(13.1)$ \\
\hline Mean general health VAS & $69.3(19.8)$ & $66.1(19.9)$ \\
\hline Mean MHI score & $71.9(20.8)$ & $70.3(20.2)$ \\
\hline \multicolumn{3}{|l|}{ Probable depression diagnosis } \\
\hline Depression & $26(25.2 \%)$ & $32(30.2 \%)$ \\
\hline No depression & 77 (74.8\%) & $74(69.8 \%)$ \\
\hline \multicolumn{3}{|c|}{$\begin{array}{l}\text { Note: Data are means (SD) or numbers }(\%) \\
B M I \text { body mass index, EQ-5DL EuroQol-5DL, HbA1c Glycated haemoglobin, } \\
\text { MHI Mental health inventory, mmol millimoles per litre, mol mole per litre, } \\
\text { PAM patient activation measure, VAS visual analogue scale }\end{array}$} \\
\hline
\end{tabular}

$\mathrm{mmol} / \mathrm{mol}$, which is equivalent to $6.2 \% \mathrm{HbA} 1 \mathrm{c}$. All participants had a BMI in the obese range $\left(>30 \mathrm{Kg} / \mathrm{m}^{2}\right)$ at baseline, with a mean BMI of 33.9 (SD 7.3). Over a quarter (28.2\%) of participants met the threshold on MHI-5 for probable depression at baseline. Participants in the two groups were similar in all respects, except that a higher proportion of females were allocated to IGR2 compared with IGR3 (48.5 vs. 39.6\%). Table 1 shows the baseline characteristics of the participants by treatment allocation.

Intervention uptake and adherence

Seven participants (one allocated to IGR2; six allocated to IGR3) withdrew before they received an action planning call. A further 10 participants (three allocated to IGR2; seven allocated to IGR3) did not receive any follow-up calls after the initial action planning call. Twenty-six participants allocated to IGR3 switched to IGR2 during the course of their exposure to the intervention. Ninetynine participants in IGR2 and 93 participants in IGR3 had at least one follow-up call and the majority of these (73\% in IGR2; $64 \%$ in IGR3) had more than five attempted follow-up calls. The mean total health advisor contact time per participant was 142.6 (SD 50.7) minutes for IGR2 and 132.2 (SD 66.5) minutes for IGR3.

\section{Outcomes}

We collected primary outcome data for 181 (87\%) participants. Missing outcome data were therefore imputed for 28 participants. At follow-up, the mean score on the CSQ-8 in IGR2 was 27.1 (SD
5.2), and in IGR3 was 28.4 (SD 4.5). Controlling for relevant covariates, the total score for the CSQ- 8 was 1.32 points higher (95\% confidence interval -0.13 to $2.77 ; p=0.074$ ) in participants allocated to IGR3 compared with those allocated to IGR2. This equates to a small effect size (standardised mean difference 0.29 , $95 \%$ C.I. -0.01 to 0.58 ). There is, therefore, weak evidence in favour of IGR3, but not at the $5 \%$ level of significance. Both the per-protocol (CSQ-8 difference $=1.33 ; 95 \%$ C.I. -0.22 to $2.88 ; p=$ 0.091 ) and complete case (CSQ-8 difference $=1.39 ; 95 \%$ C.I. -0.03 to $2.88 ; p=0.062$ ) analyses for the primary outcome returned very similar results to the intention to treat analyses: differences in total satisfaction scores between IGR2 and IGR3 were not significant at the $5 \%$ level.

Secondary outcomes were only analysed and presented for complete cases (Table 2). While secondary outcomes broadly favoured IGR3 the differences did not reach significance. Only the between group difference in BMI was borderline significant ( $p=$ 0.05).

Routine service level data

None available.

Qualitative data

To be reported in a separate process evaluation.

\section{Economic analysis}

Intervention delivery and costs. The resources needed to deliver IGR3 over the 9-month observation period included training Care Call staff and monthly IT costs (Table 3). Pedometers cost $£ 2.20$ per participant in IGR3. All participants received the Leicester Diabetes booklet at a cost of $£ 1$ per participant. Supervision of health coaching staff at Care Call cost $£ 200$ per participant over 9-months based on salary rates for $240 \mathrm{~h}$ for an Agenda for Change Band 7 manager and $1283 \mathrm{~h}$ for an Agenda for Change Band 2 administrator. The average fixed costs per participant were $£ 201$ in IGR2 and $£ 439$ in IGR3.

The average call time (including administration) for action planning was slightly higher in IGR3 compared with IGR2. Among those who had at least one attempted follow-up call the average call time (including administration) was similar in both groups (Table 4). Combining the call time (including administration) with unit costs of staff delivering the interventions, the mean cost of calls per participant was $£ 144$ (SD $£ 44$ ) for IGR2 and $£ 142$ (SD $£ 56$ ) for IGR3, excluding the seven withdrawals before action planning call attempts.

Cost effectiveness analysis with imputation. Table 5 presents the adjusted estimates of the incremental costs associated with delivery of interventions from a CCG perspective. IGR3 is associated with a mean incremental cost of $£ 236(95 \% \mathrm{Cl} £ 223$ to $£ 250)$ and a mean ICER of $£ 182(95 \% \mathrm{Cl} £ 84$ to $£ 931)$ per point improvement on the CSQ-8. The complete cases analysis showed a similar incremental costs per participant with a smaller difference in CSQ-8, leading to a much higher ICER with wider confidence interval (supplementary material 1).

\section{DISCUSSION}

There was no evidence that participants who used a digitallyenabled telephone coachin intervention that included an online dashboard plus telephone health coaching for preventing diabetes were more satisfied with their healthcare than participants who used a telephone only health coaching intervention. While there was an indication that participants who received the digital intervention were more activated, had improved health status and reduced depressive symptoms, the differences 
P. Coventry et al.

Table 2. Complete case analyses of secondary outcomes at 9-months follow-up

\begin{tabular}{|c|c|c|c|c|c|c|}
\hline Outcome & \multicolumn{2}{|l|}{ IGR2 } & \multicolumn{4}{|l|}{ IGR3 } \\
\hline $\mathrm{HbA} 1 \mathrm{c}(\mathrm{mmol} / \mathrm{mol})$ & 77 & $43.4(4.2)$ & 70 & $42.9(3.2)$ & $-0.37(-1.48$ to 0.67$)$ & 0.50 \\
\hline BMI $\left(\mathrm{kg} / \mathrm{m}^{2}\right)$ & 89 & $33.8(6.7)$ & 84 & $32.4(7.3)$ & $-0.52(-1.05$ to -0.00$)$ & 0.05 \\
\hline$E Q-5 D-5 L$ & 53 & $0.72(0.3)$ & 58 & $0.73(0.2)$ & $0.02(-0.00$ to 0.03$)$ & 0.07 \\
\hline General Health VAS $(0-100)$ & 96 & $71.5(19.4)$ & 84 & $73.3(22.1)$ & $2.86(-2.5$ to 7.9$)$ & 0.27 \\
\hline \multicolumn{7}{|l|}{ SDSCA } \\
\hline $\begin{array}{l}\text { Days ate } \geq 5 \text { portions of Fruit and } \\
\text { vegetables }\end{array}$ & 95 & $4.54(1.7)$ & 85 & $4.33(1.9)$ & & \\
\hline Days ate high fat foods & 96 & $4.35(1.8)$ & 85 & $4.26(2.1)$ & & \\
\hline $\begin{array}{l}\text { Days participated in } \geq 30 \text { mins } \\
\text { physical activity }\end{array}$ & 102 & $4.1(2.2)$ & 85 & $4.6(2.1)$ & & \\
\hline Cigarettes per day & 18 & $13.6(7.6)$ & 17 & $13.0(7.8)$ & & \\
\hline
\end{tabular}

$B M /$ body mass index, EQ-5DLEuroQol-5DL, HbA1cglycated haemoglobin, MH/mental health inventory, mmolmillimoles per litre, molmole per litre, PAMpatient activation measure, SDSCAsummary of diabetes self-care activities, VASvisual analogue scale

aAdjusted differences are those reported by the regression models - the average between-group difference, but adjusted for covariates in the model

\begin{tabular}{|lll}
\hline \multicolumn{2}{l}{ Table 3. Costs of training and online dashboard to support delivery of } \\
IGR3 & $N$ & Cost \\
\hline Hitachi training (×3 2-h sessions) & & \\
\hline Trainers & 1 & $£ 300$ \\
IT project manager & 1 & $£ 225$ \\
IT technical manager & 1 & $£ 375$ \\
Service designer & & \\
Trainees & 1 & $£ 312$ \\
Care call service manager & 6 & $£ 1512$ \\
Diabetes specialist nurse & 5 & $£ 900$ \\
Health advisor & 1 & $£ 138$ \\
Programme administrator & $£ 3,762$ \\
Total training cost & $£ 36$ \\
Training cost per participant in IGR3 & \\
Online dashboard costs (per month, including VAT) & \\
Set up and hosting services & $£ 930$ \\
Server rental & $£ 410$ \\
Third line support & $£ 1020$ \\
Total costs over 9 month trial & $£ 21,240$ \\
Dashboard cost per participant in IGR3 & $£ 200$ \\
\hline
\end{tabular}

compared with participants in the telephone only group were not significant. At follow-up participants in both groups had lower $\mathrm{HbA1C}$ and BMI but the between group difference for these outcomes was not significant. There were no significant differences between the groups for other secondary outcomes. Attrition was slightly higher in IGR3 and a quarter of participants in this group switched to IGR2 and received the telephone only intervention. However the per protocol analysis returned similar results to the intention-to-treat analysis suggesting that participants were satisfied with the overall health coaching approach regardless of allocation. Contact time between health coaches and
Table 4. Mean time in minutes for delivery of action planning and follow-up calls over 9-months by allocation group

\begin{tabular}{|c|c|c|c|c|}
\hline & \multicolumn{2}{|c|}{ IGR2 $(n=99)$} & \multicolumn{2}{|c|}{ IGR3 $(n=93)$} \\
\hline & Mean (SD) & Range & Mean (SD) & Range \\
\hline \multicolumn{5}{|c|}{ Action planning call } \\
\hline Pre-call admin & $3.8(2.8)$ & $0-15$ & $5.6(3.9)$ & $0-24$ \\
\hline Call time & $28.1(8.1)$ & $2-47$ & $28.3(11.0)$ & $5-88$ \\
\hline Post call admin & $20.1(7.4)$ & $6-45$ & $21.0(11.5)$ & $4-76$ \\
\hline Total & $52.5(13.7)$ & $13-96$ & $54.9(20.7)$ & $14-136$ \\
\hline \multicolumn{5}{|l|}{ Follow-up calls } \\
\hline Pre-call admin & $6.6(3.8)$ & $0.7-20.0$ & $6.8(4.1)$ & $0.0-20.0$ \\
\hline Call time & $21.4(5.4)$ & $11.0-45.5$ & $21.9(6.5)$ & $4.0-50.0$ \\
\hline Post call admin & $12.4(3.8)$ & $5.7-28.0$ & $13.0(5.2)$ & $5.7-40.0$ \\
\hline Total & $40.3(10.2)$ & $25.0-78.0$ & $41.8(11.4)$ & $20.0-89.0$ \\
\hline
\end{tabular}

participants was broadly equivalent in both groups. Although both were used for less than the maximum of $180 \mathrm{~h}$ allotted for the IGR pathway, IGR3 was not found to improve staff efficiency as expected. From a provider perspective the additional cost of the digital intervention was $£ 236$ ( $\$ 332 ; € 270)$ per participant to deliver, which consisted mostly of the training and platform costs of the online dashboard.

Previously it has been shown that satisfaction with telephone only health coaching is associated with higher levels of activation and number of sessions completed among those enrolled in a behaviour change programme to prevent diabetes. ${ }^{24}$ While there was a signal that participants in IGR3 were more satisfied and more activated than those in IGR2, we cannot be certain this was associated with the availability of the digitally-enabled intervention. Indeed, because there was no difference in uptake and number of follow-up telephone coaching calls between the two 
Table 5. Cost-effectiveness analysis from clinical commissioning group perspective

\begin{tabular}{|c|c|c|}
\hline Costs/outcomes & IGR2 $(N=103)$ & IGR3 $(N=106)$ \\
\hline Cost of calls made within 9 months period (mean, SE) & $£ 144(£ 4)$ & $£ 141(£ 6)$ \\
\hline Unadjusted difference in intervention costs (mean, $95 \% \mathrm{Cl}$ ) & $£ 236(£ 222-£ 250))$ & \\
\hline Adjusted difference in intervention costs ${ }^{\mathrm{a}}$ (mean, $95 \% \mathrm{Cl}$ ) & $£ 236(£ 223-£ 250)$ & \\
\hline Adjusted difference in CSQ-8 score ${ }^{\mathrm{a}}$ (mean, 95\% Cl) & $1.3(0.1-2.5)$ & \\
\hline ICER (mean, 95\% Cl) & \multicolumn{2}{|c|}{$£ 182$ (£84 - £931) per point improvement on CSQ-8 } \\
\hline
\end{tabular}

groups, it is still possible that any difference in satisfaction and activation may be attributed to the quality of health coaching.

While this trial was not set-up as a superiority test for clinical outcomes, it is instructive to compare before and after results achieved in CATFISH with those reported in comparable studies that tested lifestyle interventions for preventing diabetes in routine practice. In updating the review by Dunkley et al, Public Health England reported a pooled reduction in $\mathrm{HbA} 1 \mathrm{c}$ of $0.07 \%$ for 11 pragmatic lifestyle interventions at 12-18 months follow-up. ${ }^{25}$ A similar result was reported at 6-months for a community based diabetes prevention programme run along pragmatic lines in the $\mathrm{NHS}^{26}$ In CATFISH the overall performance of the IGR Care Call intervention was associated with a mean reduction in $\mathrm{HbA} 1 \mathrm{c}$ of $0.10 \%$ with no difference between trial groups. This is equivalent to results of more intensive national diabetes prevention programmes such as those run in the United States which showed a mean reduction in $\mathrm{HbA} 1 \mathrm{c}$ of $0.10 \%$ at 12 months. ${ }^{8}$ Similarly, weight loss results for the IGR Care Call interventions were equivalent or outperformed results from comparable pragmatic interventions such as Let's Prevent which reported a mean difference of 0.11 in $\mathrm{BMI}$ scores between the intervention group and standard care. ${ }^{26}$ Larger effects were reported by Block et al who tested the effects of a fully automated digital behaviour change intervention among people with prediabetes. ${ }^{19}$ In that trial participants who used the web-based intervention reported a mean reduction in $\mathrm{HbA} 1 \mathrm{c}$ of $0.26 \%$ and a mean reduction of 1.05 in BMI scores at 6-months. While participants were not supported by face-to-face or telephone coaching they did have access to social support via online networks and engaged in team based competitions suggesting that peer support and gamification might be critical to the success of online interventions.

Attrition is typically higher in trials of web-based interventions compared with controls, with drop-outs reported to be $22.5 \% .{ }^{27}$ In CATFISH drop out from the web plus telephone group was $24.5 \%$ when withdrawals and those who switched to the telephone only group are combined. It is clear that therapeutic contact on the telephone can improve usage and outcomes of web-based interventions. ${ }^{16}$ However, unlike other web delivered lifestyle interventions that used telephone support we did not see a difference between the two groups in uptake of the telephone element of health coaching and we do not know if telephone coaching improved usage of the digital content of the intervention. $^{28}$

This was not a cost-effectiveness study but we did assess costs attributed to the set-up and delivery of the digital intervention. The higher cost for using IGR3 were mainly associated with the web elements. The expected effect on efficiency was not found in the trial, which could be a result of lack of integration between the telephone and digital components. If the digital platform only served as a notebook, the platform could potentially only add additional burden associated with the training without any intended benefit. With no efficiency savings gained, it would be for the provider to judge if the additional cost per point of improvement on the CSQ-8 is worth paying. A key advantage of digitally-enabled interventions is that they have potential to reach economies of scale, which might take place as the user base grows. Based on modelling used in the digital pilot of the National Diabetes Prevention Programme in the $\mathrm{NHS}^{29}$ the digitallyenabled telehealth intervention designed by Hitachi is purported to achieve efficiencies of scaling over telephone only interventions at 3000 users per year with the use of cloud based infrastructure and improved management capacity. In that model the cost of the digitally-enabled intervention would be $£ 383$ per person; a fully optimised cost basis is achievable at 10000 users per year costing $£ 309$ per person. There is potential to reduce the cost of the digitally-enabled intervention further with the use of a dynamic care pathway whereby users are segmented into three categories of health coaching support. In this dynamic model the cost per person is proposed to be $£ 326$ with a user base of 3000 per year, and $£ 292$ per person with a user base of 10000 .

The digital intervention was embedded within an existing service that was delivered as part of routine care for people with $\mathrm{NDH}$ and in this sense the CATFISH trial responds to the need to address the translational gap between evidence and practice in diabetes prevention. ${ }^{30}$ Additionally the CATFISH trial evaluated end-user satisfaction as a primary outcome and thereby underscores the importance of assessing patient experience of interventions. ${ }^{31}$ As with most trials there is the possibility that we only reached and included a self-selecting cohort of eligible patients and that this group were the most motivated to engage with the intervention. However, participants were blind to treatment allocation and were not aware which intervention was novel; researchers and analysts were also blind to treatment allocation. Finally, drop outs from IGR3, including those who switched to IGR2, was moderately high but we do not know the reasons for this, nor do we know why once engaged in health coaching participants completed their call schedules. These questions will partly be addressed in a separate qualitative process evaluation.

In the context of continued roll out of diabetes prevention programmes, such as that launched by NHS England, it is important to recognise that relatively low intensity interventions such as the ones deployed in CATFISH have an important role to play in translating evidence into practice. ${ }^{32}$ Encouraging patients to engage with digital health interventions is a challenge and their reach and scaling are likely to hinge on successful outreach and enrolment activities. ${ }^{33}$ Where digital interventions are preferred it is critical that inclusion is enhanced to contend with higher rates 
of digital exclusion among the most deprived and among those who lack the confidence and skills to use online approaches. ${ }^{34}$ As with other digital interventions there is also need to consider how to maximise adherence and optimise usage and the use of games and SMS alerts, as well as improving accessibility on mobile platforms are possible avenues for further evaluation. Critical too is the need for a better understanding of the optimal combinations of behaviour change techniques and mode of delivery for web-based interventions. A review of 52 online health interventions showed that only usability was associated with effectiveness, but there was little evidence that pointed to the most effective combinations of behaviour change techniques. ${ }^{35}$ Component network meta-analysis is a relatively new approach to metaanalysis that can unpick the treatment effects of different components includes in complex composite interventions. ${ }^{36}$ There is scope for further elaboration of the most effective combinations of components for web-based behaviour change interventions using this approach. Definitive testing of candidate interventions would further enhance the evidence base for web-based behaviour change and health promotion interventions.

We showed that user satisfaction and experience of a digitalenabled telephone coaching intervention that included an online dashboard and telephone health coaching was broadly equivalent to that of a routinely delivered telephone-only coaching intervention for people with $\mathrm{NDH}$. On the basis of user satisfaction there is scope to consider this digitally-enabled telephone coaching intervention as an option for decision makers with responsibility for commissioning diabetes prevention services. However there is still uncertainty about cost-effectiveness of this digitally-enabled telephone coaching intervention and further definitive evaluation is warranted. Furthermore, future work is needed to assess whether economies of scale are achievable outside the context of trial based evaluations where user bases are likely to be much larger.

\section{METHODS}

Study design and participants

CATFISH was an individually randomised controlled trial conducted at the Salford NDH Care Call (previously known as IGR Care Call) service provided by Salford Royal NHS Foundation Trust, Greater Manchester. The trial protocol has been previously published. ${ }^{37}$ Ethical approval was granted by NHS Research Ethics Committee for the East of England (Cambridgeshire and Hertfordshire) (reference no 15/EE/0117).

Participants for CATFISH were identified from referrals from primary care or community teams to NDH Care Call. Referral criteria to NDH Care Call were: moderate or high risk on the Leicester Diabetes Risk Score Assessment $\mathrm{Tool}^{38}$ and $\mathrm{HbA} 1 \mathrm{c}=42-47 \mathrm{mmol} / \mathrm{mol}(6.0-6.4 \%)$ or a previous diagnosis of IGR with $1 \mathrm{x}$ confirmatory blood test $(\mathrm{HbA} 1 \mathrm{c}$ within the previous 6 months).

Additional eligibility criteria for inclusion in CATFISH were adults aged $>18$ years and access to a telephone and home internet. We excluded people with a diagnosis of type 2 diabetes $(\mathrm{HbA} 1 \mathrm{c}$ of $\geq 48 \mathrm{mmol} / \mathrm{mol}$ [ $\geq 6.5 \%]$ ); a diagnosis of gestational diabetes; did not read or speak English; and those incapable of participating as indicated by their GP because of dementia, learning difficulties, vision or motor skills limitations, serious and enduring mental health problems.

\section{Randomisation and masking}

Contact details of eligible patients were forwarded to the Care Call programme administrator who asked whether patients were willing to discuss participation in the CATFISH trial. Patients who verbally consented to be contacted about CATFISH were then telephoned by the research team ( $A B$ and $E B$ ) who checked their eligibility for the trial and invited them to agree to a baseline assessment. Once signed consent forms had been received and the baseline assessment had been completed participants were randomised using a central randomisation service provided by the Manchester Academic Health Science Clinical Trials Unit at the Christie NHS Foundation Trust. Participants were allocated 1:1 by minimisation using $\mathrm{BMI}$ and $\mathrm{HbA1c}$ at baseline. This technique ensures that treatment groups are very closely similar for several variables, even in small samples. ${ }^{39}$ The principal investigator and NDH programme administrator were aware of allocations; research staff and analysts were blinded to allocation. Treatment allocation was concealed from participants at the baseline assessment appointment. It was not possible to blind the health professionals delivering the interventions.

\section{Interventions}

The telephone only service was known as IGR2. The digitally-enabled telephone service was known as IGR3. The service specification was adapted from the Diabetes Care Call service and a previously piloted 6 month telephone delivered lifestyle and education programme for people with impaired glucose tolerance. ${ }^{12,40,41}$ The core content for both IGR2 and IGR3 includes education about reducing the risk of developing diabetes, goal setting and action planning, and coaching from health advisors to support patients to achieve their goals and to maintain and review their action plans. Health advisors are NHS Agenda for Change band 4 workers who received at least two months in-house induction training that included the X-pert 6-week diabetes patient education programme which includes education sessions about the prevention of diabetes, and education sessions for newly diagnosed type 2 diabetes patients and insulin starters. ${ }^{42}$ Additionally all advisors completed a 1-day training course in motivational interviewing provided by Advancing Quality Alliance and delivered by an independent training company. ${ }^{43}$ Advisors were also given educational materials, took part in telephone role-play calls, observed diabetes clinics, attended medication training with the Diabetes Team, and shadowed calls from trained advisors.

The telephone schedule was similar for IGR2 and IGR3 and comprised an introductory call from the programme administrator, followed by a 30-40 min action planning phone call from a diabetes specialist nurse or dietician [call 1]; $6 \times 10-20$ min phone calls over six months from the health advisor [calls 2-7]; and a final step-down $10-20 \mathrm{~min}$ call at nine months [call 8]. All calls were outbound and heath advisors maintained contact with the same patients throughout the care pathway.

Before the action planning call all participants received a copy of the Leicester diabetes booklet. Participants completed 'My Plan' which helped them to reflect on how they might make changes to their food choices and activity to manage their risk of diabetes. The action planning call was not scripted and focused on explaining the significance of blood tests results, understanding risk factors for diabetes, exploring participants' readiness to change and encouraging them to identify SMART goals. Weight loss targets of 5 and $10 \%$ were communicated to the participants. The six follow-up calls were guided by scripts modelled on those used by Diabetes Care Call but with an emphasis on diet and exercise and tailoring to ensure that call content was patient-centred and flexible to support their individual goals. The step-down call at 9 months provided an opportunity to negotiate and agree onward action plans and discharge participants back to GP care.

The critical innovation in IGR3 was the addition of an online dashboard that was accessible by participants and health advisors. The dashboard was co-designed with patient groups and front-line NHS Health Advisors, based on both Hitachi's experience of delivering diabetes prevention and wellbeing programmes in Japan and SRFT experience of developing and delivering a telehealth diabetes prevention programme known as Diabetes Care Call. ${ }^{12}$ The dashboard was designed for use on desktop computers only. The patient view included an interactive and dynamic log that enabled participants to track their progress against action plans and monitor their weight, physical activity, and blood glucose. Additionally the dashboard view included a space for participants to message health advisors and a progress tracker to identify completed and future call events (Supplementary 2).

Before the action planning call, the participants allocated to IGR3 were sent a pedometer to log their activity during the six months exposure to the intervention. Participants allocated to IGR3 also completed an online self-assessment before the action planning call. The IGR3 care pathway was supported by three embedded videos on the online dashboard: Introduction; Guide to Self-Assessment; Guide to the Dashboard (supplementary 3). Health advisors could access logged information to assess the progress of the participants and tailor the follow-up calls accordingly.

\section{Primary outcome}

The primary outcome was the mean difference in total score of the Client Satisfaction Questionnaire (CSQ-8) ${ }^{44}$ at 9 months post-randomisation. This 
end point was chosen to coincide with the timing of the scheduled stepdown call. The CSQ-8 is an 8-item generic survey instrument used in primary care clinical trials and has been widely used as a measure of user satisfaction of web-based interventions. ${ }^{45}$ It is scored using a four-point Likert with a range from 8 to 32; higher scores indicate higher satisfaction. The CSQ-8 was measured only at follow-up with computed scores used to calculate the mean difference between groups.

\section{Secondary outcomes}

HbA1c concentration. Glycated haemoglobin ( $\mathrm{HbA1c}$ ) was measured with a blood test taken from participants after the step-down call at the Barnes Clinical Research Facility, SRFT. Sample type and volume used were fluoride oxalate (yellow), $1 \mathrm{~mL}$; reference range and units were 3.0 to $6.0 \mathrm{mmol} / \mathrm{L}$. Participants who declined a blood test at follow-up were asked to consent to sharing the results of routine blood tests taken at their general practice. $\mathrm{HbA} 1 \mathrm{c}$ represents average blood glucose over a 8 to 12 week period. ${ }^{46}$ We used routine test results that were reported between 12 weeks before or 12 weeks after the end of follow-up.

BMI. BMI $\left(\mathrm{kg} / \mathrm{m}^{2}\right)$ was measured using height $(\mathrm{m})$ taken at baseline and weight $(\mathrm{kg})$ measured at follow-up using ISO 9001:2008 standard scales (Seca model 8751) that were calibrated using weights traceable to the National Physics Laboratory Standards of Mass.

Quality of life. The five-item EQ-5D-5L is a generic measure of healthrelated quality of life consisting of the EQ-5D descriptive system and EQ Visual Analogue Scale (EQ VAS). ${ }^{47}$ The first part consists of 5 domains: mobility, self-management, ability to do usual activities, pain, anxiety and depression, with 5 levels of severity for each domain (no, slight, moderate, severe, and extreme problems). The VAS records an individual's perceived self-rated health, ranging from 0 to 100; higher values indicate better general health. The EQ-5D-5L was used at baseline and follow-up.

Mental health. The Mental Health Inventory (MHI-5) is a 5-item scale which measures general mental health, including depression, anxiety, behavioural-emotional control and general positive affect. ${ }^{48}$ We used the recommended score $\leq 60$ (high scores on this scale represent greater wellbeing) to indicate the presence of 'probable depression'; ${ }^{49}$ continuous scores were used in the analysis. The MHI- 5 was collected at baseline and at follow-up.

Health experience and self-management. We evaluated self-management behaviours using the revised version of the Summary of Diabetes Self-Care Activities (SDSCA) scale. ${ }^{50}$ The SDSCA includes items that assess diabetes self-management across core domains related to: general diet, specific diet, exercise, blood-glucose testing, foot care, and smoking. We used items relevant to diabetes prevention and assessed the number of days per week participants engaged in healthy and unhealthy behaviours (i.e., eating $\geq 5$ portions of fruit and vegetables; eating high fat food; participating in $\geq 30 \mathrm{~min}$ of physical activity; participating in specific a specific exercise session; drinking alcohol). We also included an item on number of cigarettes smoked per day. Health experience and selfmanagement data were collected at baseline and at follow-up.

Patient activation. The Patient Activation Measure (PAM)-13 is a selfreport measure of patient knowledge, skills and confidence in selfmanagement for long-term conditions. ${ }^{51}$ We used the short 13 item version. ${ }^{52}$ The score $(0-100)$ can be used to segment patient populations into four levels of activation but we used the continuous score in the analysis. The PAM-13 was collected at baseline and at follow-up.

\section{Statistical analysis}

We powered the study to have $90 \%$ power (with a 2-sided alpha level of $5 \%$ ) to detect a standardised effect size of 0.5 on the CSQ-8. Allowing for a $15 \%$ attrition rate, 200 participants were needed in total (100 per group). Basic summary statistics (mean, SD, minimum, maximum) were calculated, by trial group, for each of our primary and secondary outcome variables. For the CSQ-8, data were only available at follow-up, whereas for all other outcomes, data were available at both baseline and follow-up. In order to formally test for post-intervention differences between CSQ-8 scores in the two groups, we conducted linear regression analysis, controlling for participant age-group, gender and baseline measurements of BMI, HbA1c, Patient Activation, MHI-5 and self-rated general health status. The primary analysis was conducted following the intention-to-treat principle. The CSQ8 was only available for 181 observations whereas between 179 and 181 observations were available for secondary outcomes. The missing data in the outcome and covariates were handled by multiple imputation using chained equations, with 20 replications. To ease interpretation and to allow comparison with published studies, we estimated a standard effect size (Cohen's d) for the CSQ-8 as the difference in follow-up means divided by the pooled baseline standard deviation for all participants. Missing data for secondary outcomes were not imputed but presented in available cases (Table 2). A per-protocol approach that analysed participants based on final treatment destination was used as a sensitivity analysis, again using the same multiple imputation approach as in the primary analysis. A secondary, complete-case, analysis was also conducted; here, we derived percentile-based, bootstrapped standard errors using 10,000 replications. Analyses were conducted using Stata Statistical Software: Release 15.

\section{Health economic analysis}

The health economic analysis aimed to identify and measure the cost and benefits of delivering IGR2 and IGR3. The primary analysis was conducted from a Clinical Commissioning Perspective (CCG) perspective using intervention costs and the CSQ-8 to derive an additional cost per additional point of the CSQ-8. CCGs are clinically-led statutory NHS bodies responsible for the planning and commissioning of health care services for their local area. All costs were presented in UK pounds Sterling $(£)$ for the financial year 2015-16. Costs were inflated to 2015-16 price levels where necessary using the Hospital and Community Health Services pay and price inflation index. ${ }^{53}$ No discount rate was applied to either costs or effectiveness because the follow-up period was 9 months.

Intervention costs. A micro-costing exercise was conducted following the methods of technology appraisal recommended by NICE. ${ }^{54}$ Training to support delivery of IGR3 was provided by Hitachi. It consisted of three 2-h sessions conducted by the IT project manager, IT technical manager and service designer. The Care Call service manager, programme administrator, six diabetes specialist nurses/diabetic dieticians and five health advisors attended the training. The cost of development of IGR3 platform was considered a 'sunk cost' and therefore not included..$^{55}$ The total costs of the training were evenly allocated to the IGR3 participants.

The online dashboard was costed for the 9 months intervention period using contract pricing provided by Hitachi. This included monthly costs for set up and hosting services, server rental and third line support, including VAT. These fixed costs were evenly allocated to each participant in IGR3.

Unit costs of staff delivering both IGR2 and IGR3 were based on estimates of costs of health and social care compiled by the Personal Social Services Research Unit (PSSRU) ${ }^{53}$ (supplementary material 4). These unit costs were then multiplied by the time spent recorded in the call log or timesheet during the trial period to estimate the costs of delivering the interventions.

Cost-effectiveness. Incremental cost-effectiveness analysis combined the intervention costs with the primary outcome to generate an incremental cost-effectiveness ratio (ICER), by dividing the mean difference in costs between the two trial groups by the mean difference in effect. ${ }^{56}$ From a CCG perspective the ICER was interpreted as the additional intervention cost per participant for one point improvement on CSQ-8.

Missing data were handled by multiple imputation following Rubin's rules, ${ }^{57}$ assuming that any missing data were missing at random. A chained equation model was developed and predictive mean matching by intervention groups was used as the imputation method, using the ten nearest neighbours to the prediction as a set to draw from. The number of imputations was set to approximately the highest percentage of missing data in all variables included in the imputation model. ${ }^{58}$ Due to the nonnormal distribution of both cost and outcome data, 5000 replacement samplings were generated using bootstrap technique to derive $95 \%$ confidence intervals. A sensitivity analysis was undertaken to repeat the CEA using complete cases.

\section{DATA AVAILABILITY}

The data that support the findings of this study are available from the corresponding author upon reasonable request. 


\section{ACKNOWLEDGEMENTS}

Thanks to Ms Charlotte Renwick for her contribution to earlier versions of the health economics analysis. We thank members of the trial steering committee for their support and critical thinking: Professor Christi Deaton, Dr Daniel Hind, and Dr Barbara Barrett. We would also like to acknowledge the support of Raj Jain, Director of Corporate Strategy, Salford Royal Foundation Trust, for continued executive sponsorship and support; Dr Sheila McCorkindale, Clinical Lead for Diabetes, Salford Clinical Commissioning Group for her unwavering support in getting CATFISH off the ground and driving the primary care referrals; Ms Jackie Steadman, Service Manager Care Call, for mobilising her team to deliver CATFISH; Ms Linda Savas, Senior Nurse Facilitator, CLARHC-GM, for her endless commitment in working with practices to identify, encourage and recruit patients to CATFISH; Ms Diane Clarke, Programme Administrator, Salford Royal Foundation Trust, for her dedication to supporting patients and the IGR Care Call team during the challenging mobilisation phase of the programme, and the IGR Care Call team at Salford Royal Foundation Trust, for their commitment and passion to supporting patients. At Hitachi we thank Adam Preis, Service Delivery Manager, for connecting stakeholders and leading the CATFISH programme across multiple organisations. Thanks to the Hitachi local staff lan Blond (business leadership), Ben Edington (technical leadership), Peter Hohmann and Giulio Moffa (design leadership), Rais Ghafoor and Riktam Bhaumik (technical management) and also to the Hitachi Limited staff based in the UK: Mr Hirohiko Hirama, Mr Yo Nakajima, Mr Atsushi Ugajin, Mr Kenichi Araki (programme leadership), Mr Koichiro Kimotsuki, Dr Shuntaro Yui and Mr Hirofumi Kondo (data science leadership), Mr Hajime Sasaki, Mr Steven Zhang, Ms Masako Hoshino, Ms Miki Kunitake. The study was funded by Hitachi Europe Ltd., and the NIHR Collaboration for Leadership in Applied Health Research and Care (CLAHRC) Greater Manchester. Hitachi played no part in the design of the study, the analysis or drafting of manuscrip. The NIHR had no role in the identification, design, conduct, or reporting of this analysis. Views expressed in this article are those of the authors and not necessarily those of the NIHR, National Health Service, or the Department of Health and Social Care.

\section{AUTHOR CONTRIBUTIONS}

Dr. P.C. (the principal investigator) and Professor P.B. were responsible for the conception and design of the study and drafted the manuscript. Dr. A.B. and Ms. E.B. were responsible for data collection, drafting the manuscript and revising it critically for intellectual content. Dr. M.H. was responsible for the statistical analysis and writing of the manuscript and revised it critically for important intellectual content. Ms. J.L. was responsible for the health economic analysis and revising the manuscript for critical intellectual content. Dr A.P. and Professor M.G. contributed to the design of the study and critical revision of the manuscript. All authors have approved the final version of this manuscript and take accountability for all aspects of the manuscript.

\section{ADDITIONAL INFORMATION}

Supplementary information accompanies the paper on the npj Digital Medicine website (https://doi.org/10.1038/s41746-019-0080-6).

Competing interests: A.P. has received a fee from Hitachi Europe Ltd. in her role as a clinical advisor. The remaining authors declare no competing interests.

Publisher's note: Springer Nature remains neutral with regard to jurisdictional claims in published maps and institutional affiliations.

\section{REFERENCES}

1. Mathers, C. D. \& Loncar, D. Projections of global mortality and burden of disease from 2002 to 2030. PLoS Med. 3, e442 (2006).

2. Zimmet, P., Alberti, K. G. \& Shaw, J. Global and societal implications of the diabetes epidemic. Nature 414, 782-787 (2001).

3. Stratton, I. M. et al. Association of glycaemia with macrovascular and microvascular complications of type 2 diabetes (UKPDS 35): prospective observational study. BMJ 321, 405-412 (2000).

4. Hex, N., Bartlett, C., Wright, D., Taylor, M. \& Varley, D. Estimating the current and future costs of Type 1 and Type 2 diabetes in the UK, including direct health costs and indirect societal and productivity costs. Diabet. Med. 29, 855-862 (2012).

5. NICE. Type 2 diabetes prevention: population and community-level interventions (PH35). https://www.nice.org.uk/guidance/ph35/resources/type-2-diabetes-preventionpopulation-and-communitylevel-interventions-pdf-1996299153349 (2011).

6. Dunkley, A. J. et al. Diabetes prevention in the real world: effectiveness of pragmatic lifestyle interventions for the prevention of type 2 diabetes and of the impact of adherence to guideline recommendations: a systematic review and meta-analysis. Diabetes Care 37, 922-933 (2014).
7. Tuomilehto, J. et al. Prevention of type 2 diabetes mellitus by changes in lifestyle among subjects with impaired glucose tolerance. N. Engl. J. Med. 344, 1343-1350 (2001).

8. Knowler, W. C. et al. Reduction in the incidence of type 2 diabetes with lifestyle intervention or metformin. N. Engl. J. Med. 346, 393-403 (2002).

9. Lawlor, M. S. et al. Cost of a group translation of the diabetes prevention program: healthy living partnerships to prevent diabetes. Am. J. Prev. Med. 44, S381-389 (2013).

10. NHS England. Next Steps on the NHS Five Year Forward View. (2017).

11. Kivela, K., Elo, S., Kyngas, H. \& Kaariainen, M. The effects of health coaching on adult patients with chronic diseases: a systematic review. Patient Educ. Couns. 97, 147-157 (2014).

12. Young, R. J. et al. Pro-active call center treatment support (PACCTS) to improve glucose control in type 2 diabetes: a randomized controlled trial. Diabetes Care 28, 278-282 (2005).

13. Sakane, N. et al. Effects of telephone-delivered lifestyle support on the development of diabetes in participants at high risk of type 2 diabetes: J-DOIT1, a pragmatic cluster randomised trial. BMJ Open 5, e007316 (2015).

14. Barak, A., Klein, B. \& Proudfoot, J. G. Defining internet-supported therapeutic interventions. Ann. Behav. Med. 38, 4-17 (2009).

15. Webb, L. T., Joseph, J., Yardley, L. \& Michie, S. Using the internet to promote health behavior change: a systematic review and meta-analysis of the impact of theoretical basis, use of behavior change techniques, and mode of delivery on efficacy. J. Med. Internet Res. 12, e4 (2010).

16. Gilbody, S. et al. Telephone-supported computerised cognitive-behavioural therapy: REEACT-2 large-scale pragmatic randomised controlled trial. Br. J. Psychiatry 210, 362-367 (2017).

17. Levine, D. M., Savarimuthu, S., Squires, A., Nicholson, J. \& Jay, M. Technologyassisted weight loss interventions in primary care: a systematic review. J. Gen. Intern. Med. 30, 107-117 (2015).

18. Watson, S. et al. Effect of a web-based behavior change program on weight loss and cardiovascular risk factors in overweight and obese adults at high risk of developing cardiovascular disease: randomized controlled trial. J. Med. Internet Res. 17, e177 (2015).

19. Block, G. et al. Diabetes prevention and weight loss with a fully automated behavioral intervention by email, web, and mobile phone: a randomized controlled trial among persons with prediabetes. J. Med. Internet Res. 17, e240 (2015).

20. Michie, S., Yardley, L., West, R., Patrick, K. \& Greaves, F. Developing and evaluating digital interventions to promote behavior change in health and health care: recommendations resulting from an international workshop. J. Med. Internet Res. 19, e232 (2017).

21. Proctor, E. et al. Outcomes for implementation research: conceptual distinctions, measurement challenges, and research agenda. Adm. Policy Ment. Health 38, 65-76 (2011).

22. Ware, J. E. Jr., Snyder, M. K., Wright, W. R. \& Davies, A. R. Defining and measuring patient satisfaction with medical care. Eval. Program Plan. 6, 247-263 (1983).

23. Boss, L. et al. Reliability and validity of assessing user satisfaction with web-based health interventions. J. Med. Internet Res. 18, e234 (2016).

24. Adams, S. R. et al. Patient satisfaction and perceived success with a telephonic health coaching program: the Natural Experiments for Translation in Diabetes (NEXT-D) Study, Northern California, 2011. Prev. Chronic Dis. 10, E179 (2013).

25. Public Health England. A systematic review of the effectiveness of lifestyle interventions for the prevention of type 2 diabetes mellitus (T2DM) in routine practice. https://assets.publishing.service.gov.uk/government/uploads/system/uploads/ attachment_data/file/733053/PHE_Evidence_Review_of_diabetes_prevention_pro grammes-_FINAL.pdf (2015).

26. Davies, M. J. et al. A community based primary prevention programme for type 2 diabetes integrating identification and lifestyle intervention for prevention: the Let's Prevent Diabetes cluster randomised controlled trial. Prev. Med. 84, 48-56 (2016).

27. Kodama, S. et al. Effect of Web-based lifestyle modification on weight control: a meta-analysis. Int. J. Obes. 36, 675-685 (2012).

28. Dennison, L. et al. Does brief telephone support improve engagement with a web-based weight management intervention?randomized controlled trial. J. Med. Internet Res. 16, e95 (2014).

29. NHS England. NHS Diabetes Prevention Programme-digital stream. https://www. england.nhs.uk/diabetes/digital-innovations-to-support-diabetes-outcomes/nhsdiabetes-prevention-programme-digital-stream/ (2019).

30. Troughton, J. et al. Development of a lifestyle intervention using the MRC framework for diabetes prevention in people with impaired glucose regulation. $J$. Public Health 38, 493-501 (2016).

31. Manary, M. P., Boulding, W., Staelin, R. \& Glickman, S. W. The patient experience and health outcomes. N. Engl. J. Med. 368, 201-203 (2013).

32. Aziz, Z., Absetz, P., Oldroyd, J., Pronk, N. P. \& Oldenburg, B. A systematic review of real-world diabetes prevention programs: learnings from the last 15 years. Implement Sci. 10, 172 (2015). 
33. O'Connor, S. et al. Barriers and facilitators to patient and public engagement and recruitment to digital health interventions: protocol of a systematic review of qualitative studies. BMJ Open 6, e010895 (2016).

34. Longley, P. A. \& Singleton, A. D. Linking social deprivation and digital exclusion in England. Urban Stud. 46, 1275-1298 (2009).

35. van Genugten, L., Dusseldorp, E., Webb, T. L. \& van Empelen, P. Which combinations of techniques and modes of delivery in internet-based interventions effectively change health behavior? A meta-analysis. J. Med. Internet Res. 18, e155 (2016).

36. Welton, N. J., Caldwell, D. M., Adamopoulos, E. \& Vedhara, K. Mixed treatment comparison meta-analysis of complex interventions: psychological interventions in coronary heart disease. Am. J. Epidemiol. 169, 1158-1165 (2009).

37. Coventry, P. A. et al. Comparison of active treatments for impaired glucose regulation: a Salford Royal Foundation Trust and Hitachi collaboration (CATFISH): study protocol for a randomized controlled trial. Trials 17, 424 (2016).

38. Gray, L. J. et al. The Leicester risk assessment score for detecting undiagnosed Type 2 diabetes and impaired glucose regulation for use in a multiethnic UK setting. Diabet. Med. 27, 887-895 (2010).

39. Altman, D. G. \& Bland, J. M. Treatment allocation by minimisation. BMJ 330, 843 (2005).

40. Betzlbacher, A. F. et al. Behaviour change among people with impaired glucose tolerance: comparison of telephone-based and face-to-face advice. J. Health Serv. Res Policy 18, 2-6 (2013).

41. Savas, L. A. et al. Prioritising prevention: implementation of IGT Care Call, a telephone based service for people at risk of developing type 2 diabetes. Prim. Care Diabetes 9, 3-8 (2015).

42. X-Pert Health. X-PERT Prevention of Diabetes (X-POD), X-PERT Diabetes and XPERT Insulin. https://www.xperthealth.org.uk/ (2019).

43. etaltraining. Motivational Interviewing Study Day. http://etaltraining.co.uk/ (2019).

44. Attkisson, C. C. \& Zwick, R. The client satisfaction questionnaire. Psychometric properties and correlations with service utilization and psychotherapy outcome. Eval. Program Plan. 5, 233-237 (1982).

45. Donker, T. et al. Internet-delivered interpersonal psychotherapy versus internet delivered cognitive behavioral therapy for adults with depressive symptoms: randomized Controlled Noninferiority Trial. J. Med. Internet Res. 15, e82 (2013).

46. Kilpatrick, E. S. Haemoglobin A1c in the diagnosis and monitoring of diabetes mellitus. J. Clin. Pathol. 61, 977-982 (2008).

47. Herdman, M. et al. Development and preliminary testing of the new five-level version of EQ-5D (EQ-5D-5L). Qual. life Res. 20, 1727-1736 (2011).

48. Berwick, D. M. et al. Performance of a five-item mental health screening test. Med. Care 29, 169-176 (1991).
49. Harrison, M. et al. A secondary analysis of the moderating effects of depression and multimorbidity on the effectiveness of a chronic disease self-management programme. Patient Educ. Couns. 87, 67-73 (2012).

50. Toobert, D. J., Hampson, S. E. \& Glasgow, R. E. The summary of diabetes self-care activities measure: results from 7 studies and a revised scale. Diabetes Care $\mathbf{2 3}$ 943-950 (2000).

51. Hibbard, J. H., Stockard, J., Mahoney, E. R. \& Tusler, M. Development of the Patient Activation Measure (PAM): conceptualizing and measuring activation in patients and consumers. Health Serv. Res. 39, 1005-1026 (2004).

52. Hibbard, J. H., Mahoney, E. R., Stockard, J. \& Tusler, M. Development and testing of a short form of the patient activation measure. Health Serv. Res 40, 1918-1930 (2005).

53. Curtis, L. Unit costs of health and social care 2016. (Personal Social Services Research Unit (PSSRU), University of Kent.(2016).

54. National Institute for Health and Care Excellence (NICE) Guide to the methods of technology appraisal 2013. http://nice.org.uk/process/pmg9 (2018).

55. McNamee, P. et al. Designing and undertaking a health economics study of digital health interventions. Am. J. Prev. Med 51, 852-860 (2016).

56. Drummond, M., Sculpher, M., Claxton, K., Stoddart, G. L. \& Geroge, W. T. Methods for the Economic Evaluation of Health Care Programmes, Fourth Edition. (Oxford University Press, Oxford, 2015).

57. Rubin, D. B. StatisticalMatching Using file concatenation with adjusted weights and multiple imputations. J. Bus. Econ. Stat. 4, 87-94 (1986).

58. White, I. R., Royston, P. \& Wood, A. M. Multiple imputation using chained equations: Issues and guidance for practice. Stat. Med. 30, 377-399 (2011).

Open Access This article is licensed under a Creative Commons Attribution 4.0 International License, which permits use, sharing, adaptation, distribution and reproduction in any medium or format, as long as you give appropriate credit to the original author(s) and the source, provide a link to the Creative Commons license, and indicate if changes were made. The images or other third party material in this article are included in the article's Creative Commons license, unless indicated otherwise in a credit line to the material. If material is not included in the article's Creative Commons license and your intended use is not permitted by statutory regulation or exceeds the permitted use, you will need to obtain permission directly from the copyright holder. To view a copy of this license, visit http://creativecommons. org/licenses/by/4.0/.

C The Author(s) 2019 